S.-B. gANK

KODAI MATH. J.

15 (1992), 165-184

\title{
ON THE FREQUENCY OF COMPLEX ZEROS OF SOLUTIONS OF CERTAIN DIFFERENTIAL EQUATIONS
}

\author{
By STEven B. BANK ${ }^{1}$
}

\begin{abstract}
.
In this paper, we investigate the frequency of zeros of solutions of linear differential equations of the form $w^{(k)}+\sum_{j=1}^{k-1} Q_{j} w^{(j)}+\left(Q_{0}+R e^{P}\right) w=0$, where $k \geqq 2$, and where $Q_{0}, \cdots, Q_{k-1}, R$ and $P$ are arbitrary polynomials with $R \not \equiv$ and $P$ non-constant. All solutions $f \not 0$ of such an equation are entire functions of infinite order of growth, but there are examples of such equations which can possess a solution whose zero-sequence has a finite exponent of convergence. In this paper, we show that unless a special relation exists between the polynomials $Q_{0}, \cdots, Q_{k-1}$, and $P$, all solutions of such an equation have an infinite exponent of convergence for their zero-sequences. This result extends earlier results for the equation, $w^{(k)}+\left(Q_{0}+R e^{P}\right) w=0$.
\end{abstract}

1. Introduction. Several recent papers (e.g. [7], [8], [9], [10], [11], [15]) have dealt with the investigation of the frequency of zeros of solutions of equations of the form,

$$
w^{(k)}+\left(R e^{P}+Q\right) w=0,
$$

where $k \geqq 2$, and where $R, P$, and $Q$ are polynomials with $R \neq 0$ and $P$ nonconstant. It was shown in $[7 ; \S 5(\mathrm{~b})$, p. 356] that for any polynomial $P(z)$ of degree $r \geqq 1$, there exists a polynomial $Q(z)$ of degree $2 r-2$ such that the second-order equation,

$$
w^{\prime \prime}+\left(e^{P}+Q\right) w=0,
$$

possesses two linearly independent solutions each having no zeros. This result led to an investigation in [8] of the more general equation (1.1) of arbitrary order $k \geqq 2$, and it was shown in [8] that if the degree of $Q$ is less than $k r-k$,

${ }^{1}$ This research was supported in part by the National Science Foundation (DMS9024930).

Received July 5, 1991. 
then the exponent of convergence (denoted $\lambda(f)$ ) of the zero-sequence of any solution $f \not 0$ of $(1.1)$ satisfies $\lambda(f)=\infty$. More recently, it has been shown in [9] that when the degree of $Q$ exceeds $k r-k$, then the conclusion $\lambda(f)=\infty$ for all solutions $f \neq 0$ of (1.1) holds except possibly when a special relation exists between $P$ and $Q$ (see $\S 4(\mathrm{~B})$ below).

To the author's knowledge, no examples have been found of an equation (1.1) of order $k>2$ which possesses a solution $f \not \equiv 0$ for which $\lambda(f)<\infty$. However, the situation concerning examples of solutions satisfying $\lambda(f)<\infty$ is far different for the broader class of equations obtained by allowing middle terms with polynomial coefficients in the equations (1.1), namely for the class of equations of the form,

$$
w^{(k)}+\sum_{j=1}^{k-1} Q_{j} w^{(j)}+\left(Q_{0}+R e^{P}\right) w=0,
$$

where $Q_{0}, \cdots, Q_{k-1}, R$ and $P$ are polynomials with $R \neq 0$ and $P$ non-constant. For example, it was shown in $[6 ;$ p. 357] that each of the third-order equations,

$$
w^{\prime \prime \prime}-w^{\prime}-e^{3 z} w=0,
$$

and

$$
w^{\prime \prime \prime}+4\left(1-z^{2}\right) w^{\prime}-\left(4 z+e^{2 z^{2}}\right) w=0
$$

possess a fundamental set of zero-free solutions. In fact, we show in $\S 9$ below that zero-free solutions of (1.3) can exist for any choice of the polynomial $P$, and can occur regardless of the order $k$.

In this paper, we investigate the frequency of zeros of solutions for the whole class of equations (1.3) of arbitrary order $k \geqq 2$. We can assume that $Q_{k-1} \equiv 0$ by the usual device of making the change of dependent variable $w=\varphi u$, where $\varphi=\exp \left(-\int\left(Q_{k-1} / k\right)\right.$, which has the effect of preserving the zero-sequence of a solution, as well as making the coefficient of $u^{(k-1)}$ equal to zero in the transformed equation. Thus, it suffices to treat the class of equations of the form,

$$
w^{(k)}+\sum_{j=1}^{k-2} Q_{j} w^{(j)}+\left(Q_{0}+R e^{P}\right) w=0,
$$

where $k \geqq 2$, and where $Q_{0}, \cdots, Q_{k-2}, R$ and $P$ arbitrary polynomials with $R \neq 0$ and $P$ non-constant. In spite of the examples (1.4), (1.5) and those constructed in $\S 9$ which have zero-free solutions, our main result ( $\$ 3$ below) shows that unless a special relation exists between the polynomials $Q_{0}, \cdots, Q_{k-2}$, and $P$ in (1.6), all solutions $f \neq 0$ of (1.6) will satisfy $\lambda(f)=\infty$. The precise form of this special relation requires certain notation from [5] which is presented in $\$ 2$ below for the reader's convenience. It should be noted that for any given equation (1.6), it is easy to check whether or not the special relation holds for the equation. We remark that the results in [8] and [9] for the equation (1.1) 
are encompassed by our main result here (see $\S 4(\mathrm{~B})$ below). In addition, our main result also sheds light on the situation for (1.1) when the degree of $Q$ equals $k r-k$, which is not treated in [8] and [9]. (See $\S 4(\mathrm{~B})$ below.)

The proof of our main result follows a pattern similar to the pattern of the proof in [9] for equation (1.1), but with additional complications. We examine the behavior of a solution $f \not \equiv 0$ of (1.6) satisfying $\lambda(f)<\infty$, in a sector where $e^{P}$ grows rapidly and in an adjoining sector where $e^{P}$ decays. Our analysis in the first sector is very similar to that in [9] for (1.1), but in the second sector is much more complicated for the following reason: In a sector where $e^{P}$ decays, the equation (1.6) can possess a property which was first investigated in [1] and is called the "global oscillation property" (see [5; p. 276]). This means that for any ray, arg $z=\theta$ lying in the sector, and for any $\varepsilon>0$, there is a solution $f \neq 0$ of (1.6) which has infinitely many zeros in the sector /arg $z-\theta \mid<\varepsilon$. (A simple example (see $\$ 7$ below) of an equation (1.6) with this property is

$$
w^{(k)}+z^{2} w^{\prime \prime}+z w^{\prime}+\left(1+e^{P}\right) w=0 \quad \text { for } \quad k \geqq 4,
$$

where $P$ is any nonconstant polynomial. A third-order example can be obtained by taking $k=3$ in (1.7) and applying the usual change of variable mentioned earlier to annihilate the second-order term.) When an equation (1.6) has the global oscillation property, a linear combination of a fundamental set in the sector need not have one term in it which asymptotically dominates the remaining terms, and the argument in [9; pp. 307-308] for (1.1) is no longer valid for (1.6). A new approach is thus required in this case, and this new approach is based on results which are proved in $\S 7$ below.

The author would like to acknowledge very valuable conversations concerning these results with his colleague, J.K. Langley.

2. Preliminaries for Main Result. Given an equation (1.6) where $Q_{0}, \cdots$, $Q_{k-2}, R$, and $P$ are polynomials, we will call the equation,

$$
w^{(k)}+\sum_{\jmath=0}^{k-2} Q_{\jmath}(z) w^{(j)}=0,
$$

the associated equation to (1.6). (The associated equation has polynomial coefficients.) We first rewrite (2.1) in tems of the operator $\theta$ which is defined by $\theta w=z w^{\prime}$. (It is easy to prove by induction that for each $m=1,2, \cdots$,

$$
w^{(m)}=z^{-m}\left(\sum_{j=1}^{m} b_{j m} \theta^{\jmath} w\right),
$$

where $\theta^{j}$ is the $j^{t h}$ iterate of the operator $\theta$, and where the $b_{j m}$ are integers with $b_{m m}=1$. In fact, as polynomials in $x$,

$$
\left.\sum_{j=1}^{n} b_{j n} x^{\jmath}=x(x-1) \cdots(x-(n-1)) .\right)
$$


When written in terms of $\theta$, let (2.1) have the form,

$$
\sum_{j=0}^{k} B_{j}(z) \theta^{\jmath} w=0, \quad \text { where } \theta^{0} w=w .
$$

(Of course, the $B_{j}(z)$ are rational functions.) By dividing the equation (2.4) through by the highest power of $z$ which occurs in all Laurent expansions of the $B_{j}(z)$ around $z=\infty$, we may assume that for each $j$, we have $B_{j}=0(1)$ as $z \rightarrow \infty$, and there exists an integer $p \geqq 0$ such that $B_{\jmath}=o(1)$ as $z \rightarrow \infty$ for $j>p$, while $B_{p}$ has a finite nonzero limit at $\infty$. As in $[5 ; \S 3]$, the integer $p$ is called the critical degree of (2.1). When (2.1) is written in the form (2.4), we then form the algebraic polynomial,

$$
H(z, v)=\sum_{j=p}^{k} z^{\jmath} B_{j}(z) v^{\jmath-p} .
$$

Then, clearly, $H(z, v)$ is a polynomial in $v$ of degree $k-p$, having rational functions for coefficients, and satisfying $H(z, 0) \not \equiv 0$. By a Newton polygon method (see $\left[14 ;\right.$ p. 105]), we determine the first terms $c z^{\beta}$ of the $k-p$ possible expansions (in descending powers of $z$ ) around $z=\infty$ of the algebraic function defined by the equation $H(z, v)=0$. The set of these first terms, $\left\{c_{1} z^{\beta_{1}}, \cdots, c_{s} z^{\beta_{s}}\right\}$ is called the exponential set for $(2.1)$ as in $[5 ; \S 3]$, and it is easy to see that $B_{j}>-1$ for each $j$. (Of course, if $k=p$ then the exponential set will be empty.)

Finally, if $g(z)$ is an analytic function on the slit plane $|\arg z|<\pi$, which has a representation of the form,

$$
g(z)=c z^{-1+d}(1+o(1)) \text { as } z \longrightarrow \infty,
$$

where $c$ is a nonzero complex number and $d$ is a positive real number, then as in $[5 ;$ p. 270], the indicial function for $g$ is defined to be the function,

$$
I F(g, \theta)=\cos (d \theta+\arg c) \text { for }-\pi<\theta \leqq \pi .
$$

3. Main Result. We now state our main result. The proof will be given in $\S 8$.

THEOREM. Given an equation (1.6) where $k \geqq 2$, and where $Q_{0}, \cdots Q_{k-2}, R$ and $P$ are any polynomials with $R \neq 0$ and $P$ non-constant. Let $\Gamma$ denote the exponential set for the associated equation to (1.6). Assume that for some real number $\theta_{0}$ in $(-\pi, \pi]$ for which $I F\left(P^{\prime}, \theta_{0}\right)=0$, the following two conditions hold (a) For any element $N$ in $\Gamma$ for which $N / P^{\prime} \rightarrow \infty$ as $z \rightarrow \infty$ in $|\arg z|<\pi$, we have $\operatorname{IF}\left(N, \theta_{0}\right) \neq 0$; (b) For any element $N$ in $\Gamma$ for which $N / P^{\prime}$ tends to a finite non-zero limit, say $c_{N}$, as $z \rightarrow \infty$ in $|\arg z|<\pi$, we have $c_{N} \neq-(k-1) / 2 k$ and

$$
I F\left(\left(c_{N}+((k-1) / 2 k)\right) P^{\prime}, \theta_{0}\right) \neq 0 .
$$

Then, the zero-sequence of any solution $f \not \equiv 0$ of (1.6) has an infinite exponent of convergence. 


\section{Remarks and Examples.}

(A) In view of example (1.4), it might be of interest to apply our result to the equation,

$$
w^{\prime \prime \prime}-w^{\prime}+R e^{P} w=0,
$$

where $R$ and $P$ are arbitrary polynomials with $R \neq 0$ and $P$ non-constant. The associated equation is $w^{\prime \prime \prime}-w^{\prime}=0$. Using (2.2) and (2.3), we write the associated equation in the form (2.4), and we obtain,

$$
z^{-3} \theta^{3} w-3 z^{-3} \theta^{2} w-\left(z^{-1}-2 z^{-3}\right) \theta w=0 .
$$

Thus the critical degree of the associated equation is $p=1$, and the algebraic polynomial (2.5) is

$$
H(z, v)=z v^{2}-3 v-\left(1-2 z^{-2}\right) z .
$$

The Newton polygon shows that the exponential set of the associated equation is $\Gamma=\left\{N_{1}, N_{2}\right\}$ where $N_{1}=1$ and $N_{2}=--1$. If the degree of $P$ is at least 2 , then $N_{j} / P^{\prime} \rightarrow 0$ for $\jmath=1,2$ as $z \rightarrow \infty$, so that the conditions (a) and (b) in the theorem are satisfied vacuously for any $\theta_{0}$ for which $I F\left(P^{\prime}, \theta_{0}\right)=0$. Thus $\lambda(f)=\infty$ for all solutions of (4.1) if $P$ has degree at least 2 .

We now assume $P(z)$ is of degree 1 , say $P(z)=r z+s$. If $r$ is real, the hypothesis (b) in the theorem is violated for the following reasons: First, the only possible values of $\theta_{0}$ are $\pm \pi / 2$. Second, the two possible values of $c_{N}$ are $\pm 1 / r$. If $r= \pm 3$, we have $c_{N}=-(k-1) / 2 k$ for one of the two elements of $\Gamma$. If $r$ is real but not \pm 3 , then (3.1) is violated at both $\theta_{0}= \pm \pi / 2$. Thus, if $r$ is real, our theorem is not applicable which is in accord with the example (1.4).

If $r$ is not real, then the theorem is applicable and we can conclude $\lambda(f)=\infty$ for all solutions $f \neq 0$ of (4.1). This can be seen as follows: Let $\theta_{0}$ be any value for which $\operatorname{IF}\left(P^{\prime}, \theta_{0}\right)=0$. It is easy to check that if $(3.1)$ is violated for either $N_{1}=1$ or $N_{2}=-1$, then $r$ would have to be real since any two zeros of the cosine must differ by a multiple of $\pi$.

(B) We remark here that for the special equation (1.1), where $k \geqq 2$ and where $R, P$, and $Q$ are polynomials, with $R \neq 0$ and $P$ of degree $r \geqq 1$, our main result encompasses the results in [8] and [9]. To see this, we note first that the equation associated to $(1.1)$ is $w^{(k)}+Q w=0$. If $Q \equiv 0$, the critical degree is $k$, so the exponential set is empty. If $Q \neq 0$, say $Q(z)=a_{n} z^{n}(1+o(1))$, then it is easy to see that the elements of $\Gamma$ are the functions $c z^{n / k}$ where $c^{k}+a_{n}=0$. Thus, if $n<k r-k$, then either $\Gamma$ is empty or each element $N$ in $\Gamma$ satisfies $N / P^{\prime} \rightarrow 0$ as $z \rightarrow \infty$ so the hypotheses (a) and (b) of our main result are satisfied vacuously. Thus we can conclude $\lambda(f)=\infty$ for all solutions if $n<k r-k$. If $n>k r-k$, then $N / P^{\prime} \rightarrow \infty$ as $z \rightarrow \infty$ for all elements $N$ in $\Gamma$. Hence, the hypothesis (b) is satisfied vacuously, and the condition given in [9] to conclude 
$\lambda(f)=\infty$ for all solutions, is precisely the condition that hypothesis (a) be satisfied for all $N$ in $\Gamma$. Of course, when $n=k r-k$ (which is the case that is not treated in [8] and [9]), we have $N / P^{\prime}$ tending to a finite non-zero limit for all $N$ in $\Gamma$, and hence for those equations (1.1) satisfying hypothesis (b), we can conclude that $\lambda(f)=\infty$ for all solutions $f \not \equiv 0$.

\section{Concepts from the Strodt theory [17].}

(a) $[17 ; \S 94]$ : The neighborhood system $F(a, b)$. Let $-\pi \leqq a<b \leqq \pi$. For each nonnegative real-valued function $g$ on $(0,(b-a) / 2)$, let $V(g)$ be the union (over all $\delta \in(0,(b-a) / 2)$ ) of all sectors, $a+\delta<\arg (z-h(\delta))<b-\delta$, where $h(\delta)=$ $g(\boldsymbol{\delta}) e^{\imath(a+b) / 2}$. The set of all $V(g)$ (for all choices of $g$ ) is denoted $F(a, b)$, and is a filter base which converges to $\infty$. Each $V(g)$ is a simply-connected region (see $[17 ; \S 93]$ ), and we require the following simple fact (see $[5 ;$ p. 269]):

LEMMA 5.1. Let $V$ be an element of $F(a, b)$, and let $\varepsilon>0$ be arbitrary. Then there is a constant $R_{0}(\varepsilon)>0$ such that $V$ contains the set, $a+\varepsilon \leqq \arg z \leqq b-$ $\varepsilon,|z| \geqq R_{0}(\varepsilon)$.

As in [2], we will say that a statement holds except in finitely many directions in $F(a, b)$, if there exist finitely many points $r_{1}<r_{2}<\cdots<r_{q}$ in $(a, b)$ such that the statement holds in each of $F\left(a, r_{1}\right), F\left(r_{1}, r_{2}\right), \cdots, F\left(r_{q}, b\right)$ separately.

(b) $[17 ; \S 13]$ : The relation of asymptotic equivalence. If $f(z)$ is an analytic function on some element of $F(a, b)$, then $f(z)$ is called admissible in $F(a, b)$. If $c$ is a complex number, then the statement $f \rightarrow c$ in $F(a, b)$ means (as is customary) that for any $\varepsilon>0$, there exists an element $V$ of $F(a, b)$ such that $|f(z)-c|<\varepsilon$ for all $z \in V$. The statement $f \ll 1$ in $F(a, b)$, means that in addition to $f \rightarrow 0$, all the functions $\theta_{\jmath}^{k} f \rightarrow 0$ in $F(a, b)$, where $\theta$, denotes the operator $\theta_{\jmath} f=z(\log z) \cdots\left(\log _{\jmath_{-1}} z\right) f^{\prime}(z)$, and where (for $\left.k \geqq 0\right), \theta_{j}^{k}$ is the $k$ th iterate of $\theta_{\jmath}$. The statements $f_{1} \ll f_{2}$ and $f_{1} \sim f_{2}$ in $F(a, b)$ mean respectively $f_{1} / f_{2} \ll 1$ and $f_{1}-f_{2} \ll f_{2}$. (This strong relation of asymptotic equivalence is designed to ensure that if $M$ is a non-constant logarithmic monomial of rank $\leqq p$ (i.e. a function of the form,

$$
M(z)=K z^{a_{0}(\log z)^{a_{1}} \ldots\left(\log _{p} z\right)^{a} p .}
$$

for real $a_{\jmath}$, and complex $K \neq 0$ ), then $f \sim M$ implies $f^{\prime} \sim M^{\prime}$ in $F(a, b)$ (see 17; $\S 28]$ ). As usual, $z^{\alpha}$ and $\log z$ will denote the principal branches of these functions on $|\arg z|<\pi)$. If $f \sim M$ in $F(a, b)$ where $M$ is given by (5.1), then we will denote $a_{0}$ by $\delta_{0}(f), a_{1}$ by $\delta_{1}(f)$ etc..

The following two facts are proved in $[12$, p. 309] and $[17 ; \S 28]$ respectively :

LEMMA 5.2. Let $f(z)$ be admissible in $F(a, b)$. Then:

(A) If $f \rightarrow 0$ in $F(a, b)$, then $z f^{\prime}(z) \rightarrow 0$ in $F(a, b)$. 
(B) If $f \ll 1$ in $F(a, b)$, then $\theta, f \ll 1$ in $F(a, b)$, for each $j=1,2, \cdots$.

We will write $f_{1} \approx f_{2}$ in $F(a, b)$ to mean that $f_{1} \sim c f_{2}$ for some nonzero constant $c$. An admissible function $f(z)$ in $F(a, b)$ is called trivial in $F(a, b)$ if $f \ll z^{-\alpha}$ in $F(a, b)$ for every $\alpha>0$. If $f \sim c z^{-1+d}$ in $F(a, b)$, where $c \neq 0$ and $d>0$, then the indicial function of $f$ is the function $\operatorname{IF}(f, \varphi)$ defined by,

$$
I F(f, \varphi)=\operatorname{Cos}(d \varphi+\arg c) \quad \text { for } \quad a<\varphi<b .
$$

(It is obvious that $I F(f, \varphi)$ has at most finitely many zeros on $(a, b)$ ). If $g$ is any admissible function in $F(a, b)$, we will denote by $\int g$, any primitive of $g$ in an element of $F(a, b)$. We will require the following two facts (see [5; p. 270]):

LEMMA 5.3. Let $f \sim c z^{-1+d}$ in $F(a, b)$, where $c \neq 0$ and $d>0$. If $\left(a_{1}, b_{1}\right)$ is any subinterval of $(a, b)$ on which $I F(f, \phi)<0$ (respectively, $I F(f, \phi)>0)$, then for all real $\alpha, \exp \int f \ll z^{\alpha}$ (respectively, $\left.\exp \int f \gg z^{\alpha}\right)$ in $F\left(a_{1}, b_{1}\right)$.

Lemma 5.4. Let $\alpha=a+b i$ be a complex number. Then for any $\varepsilon>0$, we have $z^{a-\varepsilon} \ll z^{\alpha}$ and $z^{\alpha} \ll z^{a+\varepsilon}$ in $F(-\pi, \pi)$.

We will also require the following facts. The first is obvious and the second follows from [17; Lemma 30]:

Lemma 5.5. (a) If $b$ is a real number, then on $|\arg z|<\pi$, we have $\left|z^{b \imath}\right| \leqq$ $e^{|b| \pi}$ and $\left|z^{b 2}\right| \geqq e^{-\left|b_{1}\right| \pi}$.

(b) If $f$ is a trivial function in $F(a, b)$, Then $f^{\prime}$ is also a trivial function in $F(a, b)$.

(c) $[17 ; \S 49]$. A logarithmic domain of rank zero (briefly, an $L D_{0}$ ) over $F(a, b)$ is a complex vector space $L$ of admissible functions in $F(a, b)$, which contains the constants, and such that any finite linear combination of elements of $L$, with coefficients which are logarithmic monomials of rank $\leqq p$ for some $p \geqq 0$, is either trivial in $F(a, b)$ or is $\sim$ to a logarithmic monomial of rank $\leqq p$ in $F(a, b)$. (Examples of such sets $L$ (where we can take $(a, b)$ to be any open subinterval of $(-\pi, \pi)$ ) are the set of all polynomials, the set of all rational functions, and the set of all rational combinations of logarithmic monomials of rank $\leqq 0$. More extensive examples can be found in $[17 ; \S \S 128,53])$.

If $f$ belongs to an $L D_{0}$ over $F(a, b)$, then in $F(a, b)$, clearly either $f$ is trivial or $f \sim c z^{\alpha}$ for some complex $c \neq 0$ and real $\alpha$ (so that $\delta_{0}(f)=\alpha$ ). If $f$ is trivial, we set $\delta_{0}(f)=-\infty$.

(d) $[3 ; \S 3]$. If $G(v)$ is a polynomial in $v$, whose coefficients belong to an $L D_{0}$ over $F(a, b)$, then a logarithmic monomial $M$ is called a critical monomial of $G$ if there exists an admissible function $h \sim M$ in $F(a, b)$ such that $G(h)$ is not $\sim G(M)$ in $F(a, b)$. The set of critical monomials of $G$ can be produced by 
using the algorithm in $[3 ; \S 26]$ which is based on a Newton polygon construction. This algorithm shows that the critical monomials are of rank $\leqq 0$. (In the special case where the coefficients of $G(v)$ are rational functions, the critical monomials are precisely the functions $c z^{\alpha}$ which form the first term of one of the expansions around $z=\infty$ of the algebraic function defined by $G(v)=0$. (This fact follows from $[3 ; \S 5(\mathrm{c})]$.$) )$

\section{A result from [2].}

Let $k$ be a positive integer, and let $\left\{R_{0}(z), \cdots, R_{k}(z)\right\}$ be contained in an $L D_{0}$ over $F(a, b)$ for some $(a, b)$ with $-\pi \leqq a<b \leqq \pi$, and assume that $R_{k}(z)$ is non-trivial (see $\S 5(\mathrm{~b}))$ in $F(a, b)$. Using (2.2), rewrite the equation,

$$
R_{k}(z) w^{(k)}+R_{k-1}(z) w^{(k-1)}+\cdots+R_{0}(z) w=0 .
$$

in the form,

$$
\sum_{j=0}^{k} B_{j}(z) \theta^{\jmath} w=0, \quad \text { where } \theta^{0} w=w \text {, and } \theta w=z w^{\prime} .
$$

By dividing equation (6.2) through by the highest power $\delta_{0}\left(B_{\jmath}\right)$ of $z$ which occurs in the expansions of all the functions $B_{j}(z)$ for all $j=0, \cdots, k$, we may assume that for each $j$, we have either $B_{j} \ll 1$ or $B, \approx 1$ in $F(a, b)$, and there exists an integer $p \geqq 0$ such that $B_{j} \ll 1$ for $j>p$, while $B_{p}$ is $\sim$ to a nonzero constant (denoted $B_{p}(\infty)$ ). The integer $p$ is called the critical degree of the equation (6.1). The equation,

$$
F^{*}(\alpha)=\sum_{j=0}^{k} B_{j}(\infty) \alpha^{j}=0,
$$

is called the critical equation of (6.1). Clearly $F^{*}(\alpha)$ is a polynomial in $\alpha$, of degree $p$, having constant coefficients. Let the distinct roots of $F^{*}(\alpha)$ be $\alpha_{0}$, $\cdots, \alpha_{r}$, with $\alpha_{q}$ having multiplicity $m_{q}$. (Thus, $\sum m_{q}=p$.) Let $M_{1}, \cdots, M_{p}$ be the $p$ distinct functions of the form $z^{\alpha} q(\log z)^{j}$ for $0 \leqq q \leqq r$, and integers $j$ satisfying $0 \leqq j \leqq m_{q}-1$. We call the set $\left\{M_{1}, \cdots, M_{p}\right\}$, the logarithmic set for (6.1). (If $p=0$, the logarithmic set is empty.) The following result was proved in $[2 ; \S 7]$ :

LEMmA 6.1. Let $k$ be a positive integer, and let $\left\{R_{0}(z), \cdots, R_{k}(z)\right\}$ be contained in an $L D_{0}$ over $F(a, b)$, and assume $R_{k}(z)$ is not trivial in $F(a, b)$. Let $p$ be the critical degree of equation (6.1) and let $\left\{M_{1}, \cdots, M_{p}\right\}$ be the logarithmic set for (6.1). Then, except in finitely many directions in $F(a, b)$, the equation (6.1) possesses admissible solutions $\varphi_{1}(z), \cdots, \varphi_{p}(z)$ such that $\varphi_{j} \sim M_{j}$ for $j=1, \cdots, p$.

Under the hypothesis and notation of Lemma 6.1 , any set $\left\{\varphi_{1}, \cdots, \varphi_{p}\right\}$ of admissible solutions of (6.1) satisfying $\varphi_{j} \sim M_{j}$ for $j=1, \cdots, p$ in some $F\left(a_{1}, b_{1}\right)$ is called a complete logarithmic set of solutions of $(6.1)$ in $F\left(a_{1}, b_{1}\right)$. (See [2; $\S 11]$.) The following fact was shown in $[2 ; \S 10]$ : 
LEMMA 6.2. Under the hypothesis of Lemma 6.1, any complete logarithmic set of solutions $\left\{\varphi_{1}, \cdots, \varphi_{p}\right\}$ of (6.1) which is admissible in $F\left(a_{1}, b_{1}\right)$ has the following property If $c_{1}, \cdots, c_{p}$ are any complex constants for which $\sum_{j=1}^{p} c_{j} \varphi_{j}$ is a trivial function in $F\left(a_{1}, b_{1}\right)$, then all $c_{\jmath}=0$.

We return now to the equation (6.1) which we assume has been written in the form (6.2), and has critical degree $p$. We form the algebraic polynomial $H(v)$ in $v$ of degree $k-p$ defined by,

$$
H(v)=\sum_{j=p}^{k} z^{j} B_{j}(z) v^{j-p} .
$$

The set of critical monomials of $H(v)$ (see $\S 5(\mathrm{~d})$ ) is called the exponentral set for (6.1). (In view of the remark in $\S 5(\mathrm{~d})$, this definition agrees with the definition of exponential set for (2.1) given in $\S 2$.) If $k=p$, the exponential set for $(6.1)$ will be empty. The algorithm in $[3 ; \S 26]$ shows that each element of the exponential set for (6.1) is of the form $c z^{\beta}$ where $\beta>-1$.

7. Main lemma on asymptotic integration. We begin with the concept of a "logarithmic differential field" which is defined in [16; p. 244].

DEFinition 7.1. Let $\Phi_{0}$ denote the set of all functions of the form $c z^{\alpha}$ for complex $c \neq 0$ and real $\alpha$. A logarithmic differential field of rank zero (briefly, an $\left.L D F_{0}\right)$ over $F(a, b)$, is a set $\Gamma_{0}$ of functions, each defined and admissible in $F(a, b)$, with the following properties: (i) $\Gamma_{0}$ is a differential field (where, as usual, we identify two elements of $\Gamma_{0}$ if they agree on an element of $F(a, b)$ ); (ii) $\Gamma_{0}$ contains $\Phi_{0}$; (iii) For every element $f$ in $\Gamma_{0}$ except zero, there exists $M$ in $\Phi_{0}$ such that $f \sim M$ over $F(a, b)$. (The simplest example of such a field over $F(-\pi, \pi)$ is the set of rational combinations of the elements of $\Phi_{0}$. This field contains the rational functions.) We remark that it follows immediately from $[18 ; \S 2.76$ and $\S 7: 2.73]$ that every $L D F_{0}$ over $F(a, b)$ is an $L D_{0}$ over $F(a, b)$, and so the concepts and results in $\S 6$ are valid for $L D F_{0}$. It also follows from $[17 ; \S 53(\mathrm{c})]$ that if $\Gamma_{0}$ is an $L D F_{0}$ over $F(a, b)$, then the set of functions of the form $f+T$, where $f$ belongs to $\Gamma_{0}$ and $T$ is trivial in $F(a, b)$, also forms an $L D_{0}$ over $F(a, b)$. The following theorem is proved in [5; Theorem 3.3]:

LEMMA 7.1. Let $k$ be a positive integer, and let $A_{0}(z), A_{1}(z), \cdots, A_{k}(z)$ be functions which belong to an $L D F_{0}$ over $F(a, b)$, and assume $A_{k}(z) \equiv 0$. Let $p$ be the critical degree of the equation,

$$
A_{k}(z) w^{(k)}+A_{k-1}(z) w^{(k-1)}+\cdots+A_{0}(z) w=0,
$$

and let $\left\{N_{1}, \cdots, N_{s}\right\}$ denote the exponential set for (7.1). Using (2.2), let (7.1) have the form $\Omega(w)=0$, where $\Omega(w)=\sum_{j=0}^{k} B_{j}(z) \theta^{j} w$, when written in terms of the operator $\theta$. Then, there exist a nonnegative integer $d$, with $s \leqq d \leqq k-p$, and $a$ set $\left\{V_{1}, \cdots, V_{d}\right\}$ of $d$ distinct functions such that all of the following hold. 
(a) For each $\mathrm{J}$, the function $V$, belongs to a logarithmic differential field of rank zero over $F(a, b)$, and there exists $n \in\{1, \cdots, s\}$ such that $V_{j} \sim N_{n}$ over $F(a, b)$.

(b) If $j \neq m$, then there exists a strictly positive real number $c=c(j, m)$ such that $V_{j}-V_{m} \approx z^{-1+c}$ over $F(a, b)$.

(c) For each $j \in\{1, \cdots, d\}$, the equation $\Omega_{j}(u)=0$, where

$$
\Omega_{j}(u)=\Omega\left(\left(\exp \int V_{\jmath}\right) u\right) /\left(\exp \int V_{\jmath}\right),
$$

has coefficients belonging to a logarithmic differential field of rank zero over $F(a, b)$, and has a strictly positive critical degree $t_{\jmath}$.

(d) $t_{1}+\cdots+t_{d}=k-p$.

Remark. The functions $V_{1}, \cdots, V_{d}$ can be explicitly calculated from the equation (7.1) (see $[5 ;$ p. 276]).

We are now ready to state and prove a result on the asymptotic integration of (1.6) in sectors where $e^{P}$ decays. We will prove the result for a more general class of equations.

LEMMA 7.2. Let $k$ be a positive integer, and let $A_{0}(z), \cdots, A_{k}(z)$ be functions which belong to an $L D F_{0}$ over $F(a, b)$. Assume $A_{k}(z) \neq 0$ and consider the equation (7.1). Let $p, N_{1}, \cdots, N_{s}, \Omega(w), V_{1}, \cdots, V_{d}, \Omega_{1}(u), \cdots, \Omega_{d}(u), t_{1}, \cdots, t_{d}$ be exactly as in the statement of Lemma 7.1. Let $G_{1}(z), \cdots, G_{k}(z)$ be any admissible functions in $F(a, b)$ which are trivial in $F(a, b)$ (see \$5(b)), and consider the equation,

$$
\sum_{j=0}^{k}\left(A_{j}(z)+G_{\jmath}(z)\right) w^{(j)}=0 .
$$

Using (2.2), let (7.3) have the form $\Lambda(w)=0$ where $\Lambda(w)=\sum_{\jmath=0}^{k} H_{\jmath}(z) \theta^{\jmath} w$, when written in terms of the operator $\theta$. For each $\jmath \in\{1, \cdots, d\}$, let $\Lambda_{j}(u)$ denote the operator,

$$
\Lambda_{\jmath}(u)=\Lambda\left(\left(\exp \int V_{\jmath}\right) u\right) /\left(\exp \int V_{\jmath}\right)
$$

Then, all of the following conclusions hold

(a) Each of the equations, $\Lambda(w)=0, \Lambda_{1}(u)=0, \cdots, \Lambda_{d}(u)=0$ has coefficients belonging to an $L D_{0}$ over $F(a, b)$.

(b) The critical degree of $\Lambda(w)=0$ is $p$, and for $j=1, \cdots, d$, the critical degree of $\Lambda_{j}(u)=0$ is $t$.

(c) Except in finitely many directions in $F(a, b)$, the following two conclusions (i) and (ii) hold:

(i) The equation $\Lambda(w)=0$ possesses a complete logarithmic set of solutions $\left\{\varphi_{1}, \cdots, \varphi_{p}\right\}$, and for each $j \in\{1, \cdots, d\}$, the equation $\Lambda_{j}(u)=0$ possesses a complete logarithmic set of solutions $\left\{\varphi_{j, 1}, \cdots, \varphi_{j, t_{j}}\right\}$;

(ii) If we set $\Delta_{0}=\left\{\varphi_{1}, \cdots, \varphi_{p}\right\}$, and 


$$
\Delta_{\jmath}=\left\{\left(\exp \int V_{j}\right) \varphi_{j, 1}, \cdots,\left(\exp \int V_{j}\right) \varphi_{j, t_{j}}\right\}
$$

for $\jmath \in\{1, \cdots, d\}$, then the set $\Delta_{0} \cup \Delta_{1} \cup \cdots \cup \Delta_{d}$ is a fundamental set of solutions of equation (7.3).

(d) If $\left(a_{1}, b_{1}\right)$ is any open subinterval of $(a, b)$ such that the elements of $\Delta_{0}, \Delta_{1}, \cdots, \Delta_{d}$ are all admissible in $F\left(a_{1}, b_{1}\right)$ and such that none of the indicial functions $\operatorname{IF}(V, \varphi)$ (for $j \in\{1, \cdots, d\}$ ) and $\operatorname{IF}\left(V,-V_{m}, \varphi\right)$ (for all $\}$ and $m$ with $\jmath \neq m)$ have any zeros on $\left(a_{1}, b_{1}\right)$, then the following is true. If $f \neq \equiv$ is any solution of (7.3) which is admissible in $F\left(a_{1}, b_{1}\right)$, then there exist a trivial function $G(z)$ in $F\left(a_{1}, b_{1}\right)$ and constants $c_{m}$ which are not all zero, such that on some element of $F\left(a_{1}, b_{1}\right)$ either

$$
f=c_{1} \varphi_{1}+\cdots+c_{p} \varphi_{p}+G
$$

or for some $n \in\{1, \cdots, d\}$,

$$
f=\left(\exp \int V_{n}\right)\left(\sum_{n=1}^{\iota_{n}} c_{m} \varphi_{n, m}+G\right)
$$

Proof. Set $\Phi(w)=\sum_{j=0}^{k} G_{j} w^{(j)}$. Using (2.2), let the equation $\Phi(w)=0$ have the form $\Psi(w)=0$, where $\Psi(w)=\sum_{j=0}^{k} E_{j} \theta^{\jmath} w$, when written in terms of the operator $\theta$. It then follows easily that

$$
\Lambda(w)=\Omega(w)+\Psi(w) \text { and } H_{\jmath}=B_{j}+E_{\jmath} \quad \text { for all } \jmath .
$$

Since all the functions $G$, are trivial in $F(a, b)$, clearly the same is true for the functions $E_{\jmath}$. (We note that the coefficients of $\Lambda(w)=0$ belong to an $L D_{0}$ over $F(a, b)$ since $\Lambda(w)=0$ is the equation (7.3) whose coefficients $A_{n}+G_{n}$ are contained in an $L D_{0}$ by the remark in Definition 7.1).

For each $j$, define $\Psi_{j}(u)$ by the formula,

$$
\Psi_{\jmath}(u)=\Psi\left(\left(\exp \int V_{\jmath}\right) u\right) /\left(\exp \int V_{\jmath}\right)
$$

and so clearly from (7.8) we have,

$$
\Lambda_{j}(u)=\Omega_{j}(u)+\Psi_{j}(u) \quad \text { for } \quad j=1, \cdots, d .
$$

Since the coefficients of $\Omega_{j}(u)$ belong to an $L D F_{0}$ while the coefficients of $\Psi_{j}(u)$ are all trivial in $F(a, b)$ (since all $E$, are trivial), it now follows as above that the coefficients of each equation $\Lambda_{j}(u)=0$ belong to an $L D_{0}$ over $F(a, b)$. This proves conclusion (a) completely.

Since the critical degree of $\Omega(w)=0$ is $p$, and since the functions $E$, are trivial, it now follows from (7.8) and the definition of critical degree that $\Lambda(w)=0$ also has critical degree $p$. Similarly, since the critical degree of $\Omega_{j}(u)=0$ is $t_{j}$, the same is true for $\Lambda_{j}(u)=0$ by (7.10) since the coefficients of $\Psi_{j}(u)$ are all trivial in $F(a, b)$. This proves conclusion (b).

Part (i) of conclusion (c) now follows from Lemma 6.1 and conclusions (a) 
and (b). The fact that the elements of $\Delta_{0}, \cdots, \Delta_{d}$ are all solutions of equations (7.3) follows from the definition of $\Lambda_{j}(u)$ in (7.4).

Now let $F\left(a_{1}, b_{1}\right)$ be any neighborhood system with $\left(a_{1}, b_{1}\right) \subseteq(a, b)$, on which the elements of $\Delta_{0}, \cdots, \Delta_{d}$ are all admissible, and such that none of the indicial functions $I F\left(V_{j}, \varphi\right)$ (for all $j$ ) and $I F\left(V_{j}-V_{m}, \varphi\right.$ ) (for $j \neq m$ ) have any zeros on $\left(a_{1}, b_{1}\right)$. (See Parts (a) and (b) of Lemma 7.1). By applying Lemma 5.3 with $f=V_{\jmath}$, and then with $f=V_{j}-V_{m}$ for $j \neq m$, it follows that the set of functions,

$$
Y=\left\{1, \exp \int V_{1}, \cdots, \exp \int V_{d}\right\}
$$

has the following property which we will refer to as Property $(*)$ : The ratio of two distinct elements of $Y$ is either trivial in $F\left(a_{1}, b_{1}\right)$ or its reciprocal is trivial in $F\left(a_{1}, b_{1}\right)$. It then follows from Lemma 5.4 that if $g \in \Delta_{n}$ and $h \in \Delta_{m}$, where $m$ and $n$ are distinct elements of $\{0,1, \cdots, d\}$, then either $g / h$ or $h / g$ is trivial in $F\left(a_{1}, b_{1}\right)$. Thus the sets $\Delta_{0}, \cdots, \Delta_{d}$ are mutually disjoint, and hence their union has precisely $k$ elements since $t_{1}+\cdots+t_{d}=k-p$ by Lemma 7.1. To prove that these $k$ solutions are linearly independent on any element of $F\left(a_{1}, b_{1}\right)$ on which they are admissible, we assume the contrary. Thus, on some element $T$ of $F\left(a_{1}, b_{1}\right)$, there is a linear combination of the union $\Delta_{0} \cup \cdots \cup \Delta_{d}$, with some nonzero coefficient, which vanishes identically. Letting $I$ denote the subset of $\{0,1, \cdots, d\}$ consisting of all $\}$ for which some element of $\Delta$, appears in the combination with a nonzero coefficient, we can write the dependence relation as $\sum_{j \in I} \sigma_{\jmath}=0$, where each $\sigma_{\jmath}$ is a linear combination of elements of $\Delta_{\jmath}$, and where some coefficient in the combination $\sigma_{\jmath}$ is nonzero. In view of Property (*) for $Y$, there exists an element $n \in I$ such that $\exp \int V_{n}$ asymptotically dominates all other $\exp \int V$, for $j \in I$ in $F\left(a_{1}, b_{1}\right)$ (where we define $V$, to be the zero function if $\jmath=0$ ). Writing the dependence relation as,

$$
\sigma_{n}=-\Sigma\left\{\sigma_{\jmath}: j \in I-\{n\}\right\},
$$

and dividing through by $\exp \int V_{n}$, the right-hand side of (7.12) becomes a trivial function in $F\left(a_{1}, b_{1}\right)$ by Property $(*)$, while the left-hand side becomes a linear combinations (with a nonzero coefficient) of a complete logarithmic set of solutions of $\Lambda_{n}(u)=0$ (or $\Lambda(w)=0$ if $n=0$ ). This is a direct contradiction of Lemma 6.2 , and thus we have shown that $\Delta_{0} \cup \cdots \cup \Delta_{d}$ is a fundamental set for (7.3). This proves conclusion (c).

To prove conclusion (d), we let $f \not \equiv 0$ be a solution of (7.3) which is admissible in $F\left(a_{1}, b_{1}\right)$. Thus by conclusion (c), there is an element $T$ of $F\left(a_{1}, b_{1}\right)$ on which $f$ can be written as a linear combination of the elements of $\Delta_{0} \cup \cdots$ $\cup \Delta_{d}$, and clearly not all coefficients in the combination can be zero. As in the proof of conclusion (c), if we let $l$ denote the subset of $\{0,1, \cdots, d\}$ consisting of all $\jmath$ for which some element of $\Delta$, appears in the combination with a nonzero coefficient, then we can write the relation as $f=\sum_{j \in I} \sigma_{\jmath}$, where $\sigma_{\jmath}$ is 
a linear combination of the elements of $\Delta_{\jmath}$, and where some coefficient in $\sigma$, is nonzero. Using Property (*) for $Y$ in (7.11), we have as before that there is an element $n \in I$ such that $\exp \int V_{n}$ asymptotically dominates all other $\exp \int V$, for $j \in I$ in $F\left(a_{1}, b_{1}\right)$ (where $V$, is defined to be the zero function if $\jmath=0$ ). Writing the relation for $f$ as

$$
f=\sigma_{n}+\sum\left\{\sigma_{\jmath}: \jmath \in I-\{n\}\right\},
$$

we see that if $n=0$, then (7.13) is of the form (7.6) where $G$ is trivial in $F\left(a_{1}, b_{1}\right)$, while if $n \neq 0$, we obtain (7.7) when we factor the term $\exp \int V_{n}$ from the right-hand side of (7.13). This proves conclusion (d).

Remark. In $\S 1$, it was stated that an equation (1.6) could have the "global oscillation property" in a sector where $e^{P}$ decays. To see this, we note that in a sector $a<\arg z<b$ where $e^{P}$ decays, the function $e^{P}$ is trivial in $F(a, b)$ by Lemma 5.3 so that (1.6) is an equation of the form (7.3) and hence we can apply Lemma 7.2 to it. Writing (1.6) in terms of the $\theta$ operator so it has the form $\Lambda(w)=0$, and letting $V_{1}, \cdots, V_{d}$ and $\Lambda_{1}(u), \cdots, \Lambda_{d}(u)$ be as in Lemma 7.2, we can assert that the equation (1.6) will have the global oscillation property if either of the following holds: (i) The critical equation of $\Lambda(\dot{w})=0$ possesses at least two distinct roots having the same real part; (ii) For some $\jmath \in\{1, \cdots, d\}$, the critical equation of $\Lambda_{j}(u)=0$ possesses at least two distinct roots having the same real part. To see this, assume that (i) holds so that by conclusion (c) of Lemma 7.2, we have that except in finitely many directions in $F(a, b)$, the equation (1.6) possesses solutions $f_{1} \sim z^{\alpha}$ and $f_{2} \sim z^{\beta}$, where $\alpha$ and $\beta$ are distinct but have the same real part. For any $F\left(a_{1}, b_{1}\right)$ where $f_{1}$ and $f_{2}$ exist, we can use [5; Lemma 7.1] to construct for any $\theta \in\left(a_{1}, b_{1}\right)$ and any $\varepsilon>0$, an appropriate linear combination of $f_{1}$ and $f_{2}$ which has infinitely may zeros on $|\arg z-\theta|<\varepsilon$. If (ii) holds, the solutions $f_{1}$ and $f_{2}$ are of the form $f_{m}=\left(\exp \int V_{\nu}\right) g_{m}$ for $m=$ 1,2 where $g_{1} \sim z^{\alpha}$ and $g_{2} \sim z^{\beta}$. We again use [5; Lemma 7.1] to construct an appropriate linear combination of $g_{1}$ and $g_{2}$ as before, say $c_{1} g_{1}+c_{2} g_{2}$, and thus $c_{1} f_{1}+c_{2} f_{2}$ will have infinitely many zeros on $|\arg z-\theta|<\varepsilon$.

For the example (1.7), when it is written in terms of $\theta$ using (2.2), it has the form (6.2) where $B_{0}=1+e^{P}, B_{1} \ll 1, B_{2} \sim 1$, and $B_{\jmath} \ll 1$ for $\jmath>2$. Thus in any $F(a, b)$ where $e^{P}$ is trivial, the critical equation (6.3) of (1.7) is $\alpha^{2}+1=0$, and so (1.7) satisfies condition (i) above and thus possesses the global oscillation property in $F(a, b)$.

We will also require the following result in the proof of our main result:

LEMMA 7.3. Let $n$ be a positive integer. Let $\psi_{1}, G, G_{1}, \cdots, G_{n}$ and $\sigma$ be admissible functions in an $F(a, b)$ such that $G, G_{1}, \cdots, G_{n}$ and $\sigma$ each $\rightarrow 0$ over $F(a, b)$ while for some nonzero constant $K$, we have $\phi_{1} \rightarrow K$ over $F(a, b)$. Let $\beta, \beta_{1}, \cdots, \beta_{n}$ be any nonzero complex numbers, and let $\lambda_{1}, \cdots, \lambda_{n}$ be $n$ distıncl nonzero real numbers. Set 


$$
H=\phi_{1}\left(\beta(1+G)+\sum_{j=1}^{n} \beta_{\jmath} z^{2 \lambda_{j}\left(1+G_{\jmath}\right)+\sigma}\right) .
$$

Then the following hold:

(a) If $n=1$, then over $F(a, b)$ we have,

$$
z H^{\prime}(z) / z^{2 \lambda_{1}} \longrightarrow K_{1} \text { where } K_{1}=K \beta_{1} i \lambda_{1} \neq 0 .
$$

(b) If $n>1$, then over $F(a, b), z H^{\prime}(z) / z^{2 \lambda_{1}}$ is of the form,

$$
\phi_{1}\left(\beta_{1} i \lambda_{1}\left(1+G_{1}\right)+\sum_{j=2}^{n} \beta_{j} i \lambda_{\jmath} z^{2\left(\lambda_{j}-\lambda_{1}\right)}\left(1+G_{\jmath}\right)+\sigma_{1}\right),
$$

where $\sigma_{1} \rightarrow 0$ over $F(a, b)$.

Proof. We differentiate (7.14) and compute $z H^{\prime}(z)$. Since $G, G, \phi_{1}-K$, and $\sigma$ all $\rightarrow 0$ over $F(a, b)$, we know by Lemma 5.2 that the same is true for $z G^{\prime}, z G_{\jmath}^{\prime}, z \sigma^{\prime}, z \psi_{1}^{\prime}$, and hence also for $z \psi_{1}^{\prime} / \phi_{1}$. Since $z^{2 \lambda_{\jmath}}$ is bounded by Lemma 5.5 , it now follows from the formula for $z H^{\prime}$ that,

$$
z H^{\prime}=\phi_{1}\left(\sum_{\jmath=1}^{n} \beta_{j} i \lambda_{j} z^{2 \lambda_{j}}\left(1+G_{\jmath}\right)+\sigma_{2}\right),
$$

where $\sigma_{2} \rightarrow 0$ over $F(a, b)$. Dividing the relation (7.17) by $z^{2 \lambda_{1}}$ and using the fact that $z^{2 \lambda_{1}}$ is bounded from below by a nonzero constant by Lemma 5.5 , we easily obtain (7.15) if $n=1$ and (7.16) if $n>1$.

Remark. Since relation (7.16) is of the same general form as (7.14) but has one fewer term in the summation, it is clear that Lemma 7.3 can be used repeatedly to reduce the summation to one term so that Part (a) of Lemma 7.3 is eventually applicable. Thus, if $H$ is given by (7.14) with $n>1$, then repeated operations of differentiation and multiplication by a complex power of $z$ will eventually yield a function which tends to a finite nonzero limit in $F(a, b)$.

8. Proof of the Main Result. We assume we are given an equation (1.6) satisfying the hypothesis of the theorem. We also assume initially that $\theta_{0} \in$ $(-\pi, \pi)$ and we will handle the case $\theta_{0}=\pi$ at the end of the proof. Since $\operatorname{IF}\left(P^{\prime}, \theta_{0}\right)=0$, we can assume without loss of generality that for some $\varepsilon_{1}>0$, we have

$$
I F\left(P^{\prime}, \theta\right)>0 \text { on }\left(\theta_{0}-\varepsilon_{1}, \theta_{0}\right) \text { and } I F\left(P^{\prime}, \theta\right)<0 \text { on }\left(\theta_{0}, \theta_{0}+\varepsilon_{1}\right)
$$

since our argument will be symmetric if we interchange the two intervals in (8.1).

To prove the theorem, we assume contrary to the conclusion that (1.6) possesses a solution $f \neq 0$ satisfying $\lambda(f)<\infty$. Using the theory of canonical products $\left[19\right.$; p. 251], we may write $f=G e^{h}$, where $G$ and $h$ are entire functions with $G$ of finite order of growth. Since $f$ solves (1.6), we obtain, 


$$
\left(h^{\prime}\right)^{k}+\Phi_{k-1}\left(h^{\prime}\right)+R e^{P}+Q_{0}=0,
$$

where $\Phi_{k-1}\left(h^{\prime}\right)$ is a differential polynomial of total degree at most $k-1$ in $h^{\prime}$, $h^{\prime \prime}, \cdots$, whose coefficients are polynomials in $G^{\prime} / G, G^{\prime \prime} / G, \cdots, G^{(k)} / G$, and $Q_{1}$, $\cdots, Q_{k-2}$, having constant coefficients, and whose terms of total degree $k-1$ are

$$
k\left(h^{\prime}\right)^{k-1}\left(G^{\prime} / G\right)+(k(k-1) / 2)\left(h^{\prime}\right)^{k-2} h^{\prime \prime} .
$$

The relation (8.2) is essentially the same relation that was obtained in [9; Formula (5.1), p. 304]. By following exactly the steps in the proof in $[9$; Formulas (5.1)-(5.21)], we determine an admissible function $W(z)$ in $F\left(\theta_{0}-\varepsilon_{2}\right.$, $\left.\theta_{0}+\varepsilon_{2}\right)$ for some $\varepsilon_{2}>0$, which has all of the following properties: (i) $W(z)$ is analytic and of finite order of growth for large $|z|$ in a sectorial region $\left|\arg z-\theta_{0}\right|<\varepsilon_{3}$ for some $\varepsilon_{3}>0$; (ii) there is a nonzero constant $J$ such that,

$$
W\left(r e^{i \theta}\right) \longrightarrow J \neq 0 \text { as } r \longrightarrow+\infty \text { for } \theta_{0}-\varepsilon_{3}<\theta<\theta_{0} .
$$

(iii) The function $W(z)$ has the form,

$$
W=\phi f D_{1} e^{(k-1) P / 2 k},
$$

where $D_{1}$ is an analytic branch on $F(-\pi, \pi)$ of the algebraic function $R^{(k-1) / 2 k}$, and where $\phi$ is an admissible function on $F(-\pi, \pi)$ which for some nonzero constant $K_{3}$ satisfies,

$$
\phi \longrightarrow K_{3} \neq 0 \text { over } F\left(\theta_{0}, \theta_{0}+\varepsilon_{2}\right) .
$$

We observe that it follows from Lemma 5.2(B) that,

$$
\text { Either } D_{1}^{\prime} / D_{1} \ll z^{-1} \text { or } D_{1}^{\prime} / D_{1} \approx z^{-1} \text { over } F(-\pi, \pi) \text {. }
$$

In addition, we observe that from property (i ) above for $W(z)$ and the PhragmenLindelöf principles $[19 ; \S \S 5.61,5.64]$, it follows easily from (8.4) that $W \rightarrow J$ as $z \rightarrow \infty$ in any closed sector $\theta_{1} \leqq \arg z \leqq \theta_{2}$, where $\theta_{1}>\theta_{0}-\varepsilon_{3}$ and $\theta_{2}<\theta_{0}$. Thus from $[17 ; \S 97]$, we can assert that

$$
W(z) \longrightarrow J \neq 0 \text { over } F\left(\theta_{0}-\varepsilon_{3}, \theta_{0}\right) .
$$

We now consider $W(z)$ on $F\left(\theta_{0}, \theta_{0}+\varepsilon_{3}\right)$. By (8.1) and Lemma 5.3, clearly $R e^{P}$ is trivial in $F\left(\theta_{0}, \theta_{0}+\varepsilon_{3}\right)$ so that (1.6) is of the form (7.3), and hence we can apply Lemma 7.2 to $(1.6)$ taking $(a, b)$ equal to $\left(\theta_{0}, \theta_{0}+\varepsilon_{3}\right)$. Clearly the hypothesis of Part (d) of Lemma 7.2 is satisfied when we take $\left(a_{1}, b_{1}\right)$ to be $\left(\theta_{0}, \theta_{0}+\varepsilon_{4}\right)$ for a sufficiently small $\varepsilon_{4}>0$, and so the solution $f$ is either of the form (7.6) or (7.7) on some element of $F\left(\theta_{0}, \theta_{0}+\varepsilon_{4}\right)$.

We assume first that $f$ has the form (7.6). Then it follows from the definition of the $\varphi$, that for some $\alpha>0$, we have $f \ll z^{\alpha}$ in $F\left(\theta_{0}, \theta_{0}+\varepsilon_{4}\right)$. Since $R(z)$ is a polynomial, clearly $D_{1} \ll z^{\alpha}$ for some $\alpha>0$, and so since $e^{(k-1) P / 2 k}$ is trivial in $F\left(\theta_{0}, \theta_{0}+\varepsilon_{4}\right)$, it follows from (8.5) and (8.6) that, 


$$
W(z) \longrightarrow 0 \text { over } F\left(\theta_{0}, \theta_{0}+\varepsilon_{4}\right) .
$$

In view of Lemma 5.1 (and the fact that $W(z)$ is of finite order on $F\left(\theta_{0}-\varepsilon_{4}\right.$, $\left.\theta_{0}+\varepsilon_{4}\right)$ ), it is now clear that (8.8) and (8.9) contradict the Phragmen-Lindelöf principle $[19 ; \S 5.64]$.

We now assume that $f$ has the form (7.7). In view of (8.5) and (8.6), there is an element of $F\left(\theta_{0}, \theta_{0}+\varepsilon_{4}\right)$ on which $W(z)$ has the form,

$$
W=\phi_{1} E \exp \int U
$$

where

$$
U=V_{n}+((k-1) / 2 k) P^{\prime}+D_{1}^{\prime} / D_{1},
$$

(and so $U$ is admissible in $F(-\pi, \pi)$ ), and where

$$
E=\sum_{m=1}^{t_{n}} c_{m} \varphi_{n, m}+G
$$

and finally where for some nonzero constant $K_{4}$,

$$
\phi_{1} \longrightarrow K_{4} \neq 0 \text { over } F\left(\theta_{0}, \theta_{0}+\varepsilon_{4}\right) .
$$

By Lemma 7.2, there is an element $N_{q}$ of the exponential set for (7.1) such that $V_{n} \sim N_{q}$ over $F(-\pi, \pi)$. But (7.1) is just the associated equation to (1.6), so by the notation in the main result, we have that $N_{q}$ belongs to $\Gamma$. We now distinguish three subcases:

Subcase $A . \quad N_{q} \ll P^{\prime}$ over $F(-\pi, \pi)$. In this case, we have from (8.7) and (8.11) that over $F(-\pi, \pi), U \sim((k-1) / 2 k) P^{\prime}$. Thus from Lemma 5.3 , we have that $\exp \int U$ is trivial in $F\left(\theta_{0}, \theta_{0}+\varepsilon_{4}\right)$, so from (8.10) we obtain (8.9) which gives the same contradiction as before.

Subcase B. $\quad N_{q} \gg P^{\prime}$ over $F(-\pi, \pi)$. Thus by (8.11) we have $U \sim N_{q}$ over $F(-\pi, \pi)$, and hence by the hypothesis of the theorem, we have

$$
I F\left(U, \theta_{0}\right) \neq 0 .
$$

If $\operatorname{IF}\left(U, \theta_{0}\right)<0$, then $\operatorname{IF}(U, \theta)<0$ on some interval $\left(\theta_{0}-\varepsilon_{5}, \theta_{0}+\varepsilon_{5}\right)$, and hence by Lemma 5.3 , we have $\exp \int U$ is trivial in $\left(\theta_{0}-\varepsilon_{5}, \theta_{0}+\varepsilon_{5}\right)$. In view of (8.10) this again yields $(8.9)$ which gives the same contradiction as before. If $I F(U$, $\left.\theta_{0}\right)>0$, then $I F(U, \theta)>0$ on some interval $\left(\theta_{0}-\varepsilon_{5}, \theta_{0}+\varepsilon_{5}\right)$, so by Lemma 5.3 ,

$$
\exp \left(-\int U\right) \text { is trivial on } F\left(\theta_{0}-\varepsilon_{5}, \theta_{0}+\varepsilon_{5}\right) \text {. }
$$

Now set, 


$$
W_{0}=W \exp \left(-\int U\right) \text { on } F\left(\theta_{0}-\varepsilon_{5}, \theta_{0}+\varepsilon_{5}\right) .
$$

Clearly $W_{0}$ is of finite order of growth on its domain. In view of (8.8) and (8.15), it follows that for all real $\alpha$, we have $z^{\alpha} W_{0} \rightarrow 0$ over $F\left(\theta_{0}-\varepsilon_{3}, \theta_{0}\right)$, and so by $[4$; Lemma 7$]$, we have

$$
W_{0} \text { is trivial in } F\left(\theta_{0}-\varepsilon_{3}, \theta_{0}\right) \text {. }
$$

Now, on $F\left(\theta_{0}, \theta_{0}+\varepsilon_{4}\right)$, we have $W_{0}=\phi_{1} E$ by (8.10), where $E$ is given by (8.12) and $\phi_{1}$ satisfies (8.13). In the expression for $E$, we know that in $F\left(\theta_{0}, \theta_{0}+\varepsilon_{4}\right)$, the function $G$ is trivial, while for each $m=1, \cdots, t_{n}$, we have

$$
\varphi_{n, m}=z^{\alpha}(\log z)^{\beta} m\left(1+L_{m}\right), \quad \text { where } L_{m} \ll 1,
$$

and where $\alpha_{m}$ is a complex number while $\beta_{m}$ is a nonnegative integer, and where the pairs $\left(\alpha_{m}, \beta_{m}\right)$ are all distinct. Let $l$ be the set of all $m$ such that $c_{m} \neq 0$ in (8.12). Write $\alpha_{m}=\sigma_{m}+i \lambda_{m}$ where $\sigma_{m}$ and $\lambda_{m}$ are real. Let $\sigma$ be the maximum of all $\sigma_{m}$ for $m \in I$, and let $I_{1}$ be the subset of $I$ consisting of those $m$ for which $\sigma_{m}=\sigma$. Let $\beta$ denote the maximum of all $\beta_{m}$ for $m \in I_{1}$, and let $I_{2}$ be the subset of $I_{1}$ consisting of those $m$ for which $\beta_{m}=\beta$. It is then easy to see (using Lemmas 5.4 and 5.5) that,

$$
E=z^{\sigma}(\log z)^{\beta}\left(\sum_{m \in I_{2}} c_{m} z^{2 \lambda_{m}}\left(1+L_{m}\right)+u_{1}\right),
$$

where $u_{1} \rightarrow 0$ over $F\left(\theta_{0}, \theta_{0}+\varepsilon_{4}\right)$. Let $I_{2}=\left\{m_{1}, \cdots, m_{s}\right\}$, and let $S=z^{i+\imath \lambda} m(\log z)^{\beta}$, where $m=m_{1}$. If $s=1$, then from (8.10), (8.13), (8.19), and Lemma 5.5, we have,

$$
W_{0} / S \longrightarrow K_{4} c_{m_{1}} \neq 0 \text { over } F\left(\theta_{0}, \theta_{0}+\varepsilon_{4}\right) \text {. }
$$

But from (8.17) and Lemma 5.4, we have the $W_{0} / S \rightarrow 0$ over $F\left(\theta_{0}-\varepsilon_{3}, \theta_{0}\right)$ and so again we have a contradiction of the Phragmen-Lindelöf principle.

If $s>1$, we have from (8.19) that

$$
W_{0} / S=\phi_{1}\left(c_{m}\left(1+L_{m}\right)+\sum_{q \in I_{2}-(m)} c_{q} z^{2\left(\lambda_{q}-\lambda_{m}\right)}\left(1+L_{q}\right)+u_{2}\right)
$$

where $u_{2} \rightarrow 0$ over $F\left(\theta_{0}, \theta_{0}+\varepsilon_{4}\right)$ by Lemma 5.5 , and where $m=m_{1}$. Noting that the numbers $\lambda_{q}-\lambda_{m}$ for $q \in I_{2}-\{m\}$ are all distinct and nonzero (since the pairs $\left(\alpha_{q}, \beta_{q}\right)$ in (8.18) are distinct), we see that (8.21) has the form (7.14) and so Lemma 7.3 is applicable. If $s=2$, then by Lemma 7.3(a), the function,

$$
W_{1}=z\left(W_{0} / S\right)^{\prime} z^{2\left(\lambda_{m}-\lambda_{r}\right)} \text {, where } r=m_{2},
$$

has the property that $W_{1}$ tends to a finite nonzero limit over $F\left(\theta_{0}, \theta_{0}+\varepsilon_{4}\right)$. However, in view of (8.17) and Lemma 5.4, clearly $W_{1} \rightarrow 0$ over $F\left(\theta_{0}-\varepsilon_{3}, \theta_{0}\right)$. This again violates the Phragmen-Lindelöf principle. (We note that $W_{1}$ is of finite order of growth over $F\left(\theta_{0}-\varepsilon_{4}, \theta_{0}+\varepsilon_{4}\right)$, since $W_{0}$ and all of its derivatives have this property by the representation for $W_{0}$ developed in $[9$; Formula 
(5.16)].) If $s>2$, then Lemma 7.3(b) applied to (8.21) shows that $W_{1}$ is given by an expression of the general form (7.16) which has one fewer term in the summation than (8.21) has. Of course, (7.16) is again of the general form (7.14), and so Lemma 7.3 can now be applied to $W_{1}$. Clearly, the process can be repeated and eventually reduces the summation to one term which results in a function which violates the Phragmen-Lindelöf principle as above. Thus Subcase $B$ is impossible.

Subcase $C$. If neither of the previous subcases hold, we must have $N_{q} \approx P^{\prime}$ over $F(-\pi, \pi)$ (see $[17 ; \S 41]$ ), say $N_{q} \sim b_{q} P^{\prime}$ where $b_{q}$ is a nonzero constant. By the hypothesis of the theorem, $b_{q} \neq-(k-1) / 2 k$, so by (8.11) and (8.7), we have

$$
U \sim\left(b_{q}+(k-1) / 2 k\right) P^{\prime} \quad \text { over } F(-\pi, \pi) .
$$

By the hypothesis (3.1) of the theorem, $I F\left(U, \theta_{0}\right) \neq 0$. This is exactly the same condition (8.14) as we had in Subcase $B$, and the proof that both possibilities, $I F\left(U, \theta_{0}\right)<0$ and $I F\left(U, \theta_{0}\right)>0$, lead to contradictions, is exactly as in Subcase $B$. Thus the proof of the theorem is complete in the case $\theta_{0} \in(-\pi, \pi)$.

In the case where $\theta_{0}=\pi$, we perform the change of variable $\zeta=-z$ in (1.6) which results in an equation which is satisfied by all functions $f(-\zeta)$ for which $f(z)$ satisfies (1.6). A routine calculation (using $[3 ; \S 26]$ ) of the exponential set of the transformed equation shows that this equation satisfies the hypotheses of the theorem for the value $\theta_{0}=0$. Thus $\lambda(g)=\infty$ for all solutions $g \neq 0$ of the transformed equation, and it follows that the same conclusion holds for the original equation (1.6).

9. Examples. In this section, we construct examples of equations (1.6) having zero-free solutions.

Example 1. Let $P(z)$ be any nonconstant polynomial, and let $K_{1}, K_{2}$, and $K_{3}$ be the cube roots of -1 . Then, the three functions,

$$
f_{j}=\exp \left((-P / 3)+\int_{0}^{z} K_{j} e^{P / 3}\right) \quad \text { for } \quad j=1,2,3,
$$

all solve the equation

$$
w^{\prime \prime \prime}+Q_{1} w^{\prime}+\left(e^{P}-\left(P^{\prime} P^{\prime \prime} / 9\right)+\left(P^{\prime \prime \prime} / 3\right)\right) w=0,
$$

where $Q_{1}=\left(2 P^{\prime \prime} / 3\right)-\left(\left(P^{\prime}\right)^{2} / 9\right)$.

This example is easily verified by routine calculation, and shows that zerofree solutions of (1.6) can occur for any choice of the polynomial $P(z)$. (The examples (1.4) and (1.5) arise by taking $P(z)=3 z+\pi i$ and $P(z)=3 z^{2}+\pi \imath$ respectively in Example 1.) The exponential set for the equation associated to (9.2) consists of two elements $N_{j}$ where $N_{j} \sim \pm P^{\prime} / 3$ over $F(-\pi, \pi)$ and hence hypo- 
thesis (b) in our theorem is violated for (9.2) since $k=3$.

Example 2. This example shows that zero-free solutions of (1.6) can occur for any order $k$. We prove :

PROPOSITION. Let $k$ be a positive integer greater than one, and let $c=-$ $(k-1) / 2$. Then, the zero-free function, $\exp \left(c z+e^{z}\right)$, solves an equation (1.6) where $Q_{0}, \cdots, Q_{k-2}$ are constants, $R \equiv-1$ and $P(z)=k z$.

Proof. Let $h(z)=c z+e^{z}$ and $f=e^{h}$. Then, it is easy to verify (e.g. see [13; Lemma 3.5]) that for each $n=1,2, \cdots$, there are constants $\beta_{n, \text {, such that }}$

$$
f^{(n)} / f=e^{n z}+\sum_{j=0}^{n-1} \beta_{n, j} e^{\jmath z} .
$$

Our choice of $c$ shows that $\beta_{k, k-1}=0$, so that

$$
e^{k_{2}}=f^{(k)} / f-\sum_{j=0}^{k-2} \beta_{k, j} e^{j z} .
$$

Thus, if $k=2$, we are done. Assuming $k>2$, we have from (9.3) for $n=k-2$,

$$
e^{(k-2) z}=f^{(k-2)} / f-\sum_{j=0}^{k-3} \beta_{k-2, .} e^{\jmath z} .
$$

We then substitute this into (9.4). In the resulting relation, we then substitute the expression for $e^{(k-3) z}$ given by (9.3) for $n=k-3$. We continue this process for $e^{(k-4) 2}, \cdots, e^{2}$, and the resulting expression is the desired equation for $f$.

\section{REFERENCES}

[1] S. BANK, A note on the location of complex zeros of solutions of linear differential equations, Complex Variables 12 (1989), 159-167. Short Versıon: Bull. Amer. Math. Soc. (New Series) 18 (1988), 35-38.

[2] S. BANK, An asymptotic analog of the Fuchs regularity theorem, J. Math. Anal. Appl. 16 (1966), 138-151.

[3] S. BANK, On the instability theory of differential polynomials, Ann. Math. Pura Appl. 74 (1966), 83-112.

[4] S. BANK, On solutions having large rate of growth for nonlinear differential equations in the complex domain, J. Math. Anal. Appl. 22 (1968), 129-143.

[5] S. BANK, On the oscillation of solutions of non-homogeneous linear differential equations, Analysis 10 (1990), 265-293.

[6] S. Bank, G. Frank and I. LaINE, Über die Nullstellen von Lösungen linearer Differentialgleichungen, Math. Zeit. 183 (1983), 355-364.

[7] S. BANk AND I. LAINe, On the oscillation theory of $f^{\prime \prime}+A f=0$ where $A$ is entire, Trans. Amer. Math. Soc. 273 (1982), 351-363.

[8] S. BANK AND J.K. LANGLEY, On the oscillation of solutions of certain linear differential equations in the complex domain, Proc. Edinburgh Math. Soc. 30 (1987), 455-469.

[9] S. BANK AND J.K. LANGLey, On the zeros of the solutions of the equation $w^{(k)}$ 
$+\left(R e^{P}+Q\right) w=0$, Kodai Math. J. 13 (1990), 298-309.

[10] S. BANK, I. LAINE AND J.K. LANGLEY, On the frequency of zeros of solutions of second-order linear differential equations, Resultate der Math. 10 (1986), 8-24.

[11] S. BANK, I. LAine AND J. K. LANGLEY, Oscillation results for solutions of linear differential equations in the complex domain, Resultate der Math. 16 (1989), 3-15.

[12] E. W. Chamberlain, The univalence of functions asymptotic to nonconstant logarithmic monomials, Proc. Amer. Math. Soc. 17 (1966), 302-309.

[13] W. K. Hayman, Meromorphic Functions, Clarendon Press, Oxford, 1964.

[14] E. Hille, Analytic Function Theory, Volume II, Chelsea, New York, (1973).

[15] J.K. LANGLEY, On complex oscillation and a problem of Ozawa, Kodai Math. J. 9 (1986), 430-439.

[16] W. STRODT, On the algebraic closure of certain partially ordered fields, Trans. Amer. Math. Soc. 105 (1962), 229-250.

[17] W. STRODT, Contributions to the asymptotic theory of ordinary differential equations in the complex domain, Mem. Amer. Math. Soc., No. 13 (1954).

[18] W. StRODT AND R.K. WRIGHT, Asymptotic behavior of solutions and adjunction fields for nonlinear first-order differential equations, Mem. Amer. Math. Soc., No. 109 (1971).

[19] E.C. Titchmarsi, Theory of Functions, Oxford University Press, London, 1939.

Department of Mathematics

UNIVERSITY OF ILLINOIS

1409 West Green Street

URBANA, ILLINOIS 61801-2975

U.S.A. 